\title{
Ionization of hydrogen and hydrogenic ions by antiprotons
}

\author{
D.R. Schultz, P.S. Krstić, and C.O. Reinhold \\ Physics Division, Oak Ridge National Laboratory, \\ Oak Ridge, TN 37831-6373 \\ J.C. Wells \\ Institute for Theoretical Atomic and Molecular Physics, \\ Harvard-Smithsonian Center for Astrophysics, Cambridge, MA 02138
}

\begin{abstract}
Presented here is a description of the ionization of hydrogen and hydrogenic ions by antiproton-impact, based on very large scale numerical solutions of the time-dependent Schrödinger equation in three spatial dimensions and on analysis of the topology of the electronic eigenenergy surfaces in the plane of complex internuclear distance. Comparison is made with other theories and very recent measurements.
\end{abstract}

34.50.Fa, 34.20.Mq, 36.10-k

Typeset using REVTEX 
Intense, well collimated, monoenergetic beams of low energy antimatter projectiles, such as positrons and antiprotons, have only recently been available for use in the study of ionatom and ion-solid interactions (see e.g. [1]). In addition to their intrinsic novelty, their utility stems from the fact that they allow one to probe the change in collision dynamics and reaction probabilities when only a single characteristic of the projectile is changed. That is, comparison of electron- and proton-impact collisions reflects a change in projectile charge sign and projectile mass simultaneously, whereas comparison of antiproton- and protonimpact isolates the differences arising from the varying charge sign. Considerable insight has been obtained in the last decade by comparing the ratio of double to single ionization [2], the spectrum of electrons ejected in ionization [3], and the variation of stopping power [4.5] (the well known Barkas effect), presented by antimatter-impact.

Prompted in large part by very recent experiments which have provided the first measurements of the single ionization cross section by antiproton-impact [6],7], presented here is a detailed description of ionization in antiproton-impact of atomic hydrogen and hydrogenic ions. The results obtained reveal a remarkable difference in the mechanisms and behavior of ionization at low collision energy. The physical picture developed is based upon solutions of the time-dependent Schrödinger equation (TDSE) on very large, three dimensional, numerical lattices, and on an analysis of the quasi-molecular electronic eigenenergy surfaces in the plane of complex internuclear distance. These methods circumvent uncertainties introduced by treating these colliding systems classically or through perturbation theory.

Antiproton collisions with atomic hydrogen and hydrogenic ions provide a unique and fundamental testing ground for the development of non-perturbative, quantal scattering techniques. Unlike proton-impact where the final state is a superposition of elastic scattering, excitation, ionization, and charge transfer, for antiproton-impact only the first three of these can be obtained. This essential difference is obviously a result of the change in the charge sign of the projectile, the negatively charged antiproton not supporting any bound electronic states.

In particular, the ionization cross section for proton-impact of atomic hydrogen displays 
a peak at an impact energy of about $50 \mathrm{keV}$ and decreases below this energy due to $(i)$ the lack of a strong coupling between the relevant quasi-molecular electronic eigenstates and the continuum, and $(i i)$ the strong coupling with the charge transfer channel. On the other hand, it has been predicted classically [8] that for antiproton-impact the cross section should not fall off in this low energy regime. Quantum mechanically, implications that such a behavior should occur are provided by the fact that all the quasi-molecular states are promoted to the continuum for small internuclear distances [9, 10]. Very recent experiments [7] have nearly reached energies sufficiently low to demonstrate (or deny) this behavior for hydrogen targets. However, an experiment employing helium targets by the same group and part of its interpretation based on perturbation theory have cast some doubt on this picture [6]. Here we provide conclusive evidence which resolves the controversy for one-electron systems.

To understand the antiproton-hydrogen system in more detail, consider the quasimolecular eigenenergy displayed as a function of distance $(R)$ between the antiproton and the proton given in Figure 1a. The character of these states is very similar to those in a dipole potential $V(\vec{r})=-\vec{d} \cdot \vec{r} / r^{3}$. In the limit of small $R$, the electron is bound to the quasi-molecule by a dipole-like potential produced by the two heavy particles of charge $-Z$ and $+Z$ where the dipole moment is $d=Z R$ and $r$ is the electronic coordinate relative to the center-of-mass. There exists a critical value $d_{c}$ below which no bound states can be supported [9:10]. Therefore, the electron cannot be bound for interparticle separations below a critical value $R_{F T}=d_{c} / Z=0.639 / Z$ a.u., known as the Fermi-Teller radius [9]. At this distance the electronic eigenenergies of $n s$-states merge with the quasi-molecular continuum edge. In contrast, the eigenenergies stay bound in the united atom limit for the proton-hydrogen system and for antiproton-hydrogenic-ion systems (Figure 1b).

The existence of this critical radius for the antiproton-hydrogen system has an immediate consequence for the behavior of the ionization cross section for collisions with small velocity, $v$. In general, when $v \rightarrow 0$, colliding systems may adiabatically deform in the course of collision, rendering inelastic transitions improbable. This system provides an exception to 
this rule because its eigenenergies are degenerate with the continuum and mutually for $R \leq R_{F T}$. More precisely, $R_{F T}$ is an essential singularity of the eigenenergy surface and for $R \leq R_{F T}$ the system becomes unstable [11], decaying into the continuum. This imposes an ideal lower bound on the low-energy ionization cross section given by $\pi R_{F T}^{2}$ a.u., assuming a straight-line approximation for the internuclear motion.

However, in reality, when $R>R_{F T}$, significant nonlocalized transitions are also expected between the ground state and the continuum through the nonadiabatic coupling operator $\partial / \partial t$ when the binding energy $E(R)$ of the initial quasi-molecular state satisfies $E(R) / v \ll$ 1 a.u.. This condition is fulfilled at a radius of about 1 a.u. for the $1 s \sigma$ state (Figure 1a) for $v \gtrsim 0.1$ a.u. which provides a practical limiting value of the cross section. Thus the adiabatic regime for the antiproton-hydrogen system is limited to collision energies near the threshold for ionization, in contrast to the proton-hydrogen system where the onset of this regime occurs at much higher energies.

This picture is supported by the present TDSE calculations, displayed in Figure 2. This method is an outgrowth of earlier pioneering studies which were restricted to smaller lattice sizes and/or fewer dimensions [12]. In the present method, the electronic wavefunction and the Hamiltonian operator are discretized on a large 3-dimensional spatial lattice of points using well known pseudo-spectral methods. The initial ground state of hydrogen evolves in time under the interaction with the projectile which moves along a classical trajectory, computed using the ground potential energy curve in order to account for possible trajectory effects. Calculating the overlap between the time-evolved state and lattice eigenstates allows the determination of reaction probabilities, and thus the ionization cross section. The use of this method in three dimensions to compute cross sections for a wide range of energies has been a significant computational challenge, and to the best of our knowledge, represents the first such application of this technique in ion-atom collisions making a quantitative comparison with experiment. The lack of charge transfer for antiproton-impact simplifies the calculation since one need not follow states bound to the projectile. The method is described in much greater detail in a subsequent publication [13]. 
At high energies (above $100 \mathrm{keV}$ ), the TDSE result is in good agreement with the experimental measurments [7], the atomic orbital close coupling treatments of Toshima [14] $\left(\mathrm{CC}_{s}\right)$, of Matir et al. [15] $\left(\mathrm{CC}_{m}\right)$, and of Schiwietz [5] $\left(\mathrm{CC}_{s}\right)$, and with the pertubative continuumdistorted-wave-eikonal-initial-state (CDW-EIS) approximation. Below this energy, the close coupling treatments $\mathrm{CC}_{t}$ and $\mathrm{CC}_{m}$ overestimate the result while $\mathrm{CC}_{s}$ follows the TDSE calculation rather closely. The CDW-EIS approximation clearly fails at energies below $50 \mathrm{keV}$. Also shown in this figure is the cross section computed with the classical trajectory Monte Carlo (CTMC) method [8,16] which gives a reasonable result over a wide range of energies. The cross section stays large at low collision energy since most of the classical orbits become unstable when the antiproton has an impact parameter smaller than the mean radius of the atom, in analogy with the quantal merging of the eigenenergies with the continuum. For larger impact parameters, the orbits adiabatically adjust to the perturbation and ionization is suppressed. At very low energies, near the ionization threshold $(27.2 \mathrm{eV})$, the polarization of the initial electronic cloud draws the antiproton in from larger impact parameters and the cross section rises. This trend is continued at even lower energies as the antiproton can no longer directly ionize the atom, but as the bound state of an antiproton and proton (protonium) is formed. This exotic capture process dominates the production of a free electron for very slow collisions.

Further, both the $\mathrm{CC}_{s}$ and TDSE results indicate a rather flat cross section between 0.2 and $100 \mathrm{keV}$, with a value in the range of approximately $1.4 \pi a . u$.. This is about a factor of three larger than the cross section predicted on the basis of the Fermi-Teller model, indicating that the excess arises due to the nonlocalized character of the transitions even in very slow antiproton-hydrogen collisions. That is, in the strict adiabatic limit, transitions are improbable except in localized [18 regions where the curves become degenerate, as in the antiproton-hydrogen case for small enough distances.

In contrast, when an antiproton collides with a hydrogenic ion $(Z>1)$ the united atom charge $Z-1$ is not equal to zero and the quasi-molecular electronic states do not become degenerate as $R \rightarrow 0$. In this case, as the collision velocity increases from zero the 
transitions are localized to points where the quasi-molecular curves cross. Since terms of the same symmetry which support radial transitions cannot cross, these points are shifted to the plane of complex $R$, and are known as hidden crossings. The most elaborate treatment of the transitions induced by these complex crossings is the so-called hidden crossings (HC) method [17] which is exact in the limit of small velocities. In this approach, the transition probability rises exponentially from zero with increasing collision velocity, the exponent being inversely proportional to $v$.

All information necessary to describe ionization in slow collisions is contained in the topology of the hidden crossings. These crossings appear as branch points of the eigenenergy surface, which we have computed for antiprotons colliding with various hydrogenic ions through numerical solution of the adiabatic Schrödinger equation. In Figure 3 we display the most important hidden crossings which lead to ionization of the ground states of these ions. They are organized in the so-called $S$-superseries, whose terms connect pairwise and in succession the $(n, s, \sigma)$ and $(n+1, s, \sigma)$ quasi-molecular states (using the spherical quantum numbers of the united atom. There is a limiting point of each superseries when $n \rightarrow \infty$ localized closely to all other points of the same superseries. This localization is described approximately by the size of the symbols in Figure 3 and their small values are the cause of a very steep diabatic promotion to the continuum from the ground state. Each of the systems presented here has one such series and therefore there is only one, strongly localized channel for ionization of a hydrogenic ion in slow collisions with antiprotons. As noted above, the exception is the antiproton-hydrogen system for which the whole superseries degenerates to the Fermi-Teller limit $\left(R_{F T}\right)$ on the real axis, which is an essential singularity rather than a branch point. Thus, the hidden crossing method is not applicable. As soon as the target nuclear charge is increased beyond one, the $S$-superseries of branch points emerges, as shown in Figure 3.

To demonstrate the behavior of ionization for antiproton-hydrogenic-ion systems, we have computed the TDSE, CTMC, CDW-EIS and $\mathrm{HC}$ result for $p^{-}+\mathrm{He}^{+}$, displayed in Figure 4, which exhibits the exponential drop of localized transitions predicted by $\mathrm{HC}$ at 
low energies. The TDSE calculations follow the $\mathrm{HC}$ results at low energies, CTMC at intermediate energies, and CDW-EIS at high energies. Evident in this figure is the upturn of the cross section at very low energies which is due to the bending of the antiproton trajectory caused by the Coulomb attraction with the $\mathrm{He}^{+}$ion. The onset of this upturn is seen at somewhat higher energies than in $p^{-}+H$ since the long ranged Coulomb interaction is stronger than the polarization interaction. A more detailed analysis of the topology of the hidden crossings for antiproton-impact of hydrogenic ions is given a forthcoming paper [19.

In brief, we note that the prediction of the $\mathrm{HC}$ method regarding this pathway to ionization has not been seen for collisions involving impact by positive ions due to two reasons. First, ionization through charge transfer channels which plays a significant role for positiveion impact does not take place. Second, the $S$-promotion mechanism described here is not the Fano-Lichten promotion mechanism which is associated with the passing of the centrifugal barrier (for antiproton-impact of target s-states such a centrifugal barrier is absent). Instead, the promotion takes place due to the repulsive potential barrier between the electron and the antiproton. Such promotion was recently reported in the context of a model of double ionization in antiproton-helium collisions [20].

Summarizing, ionization of atomic hydrogen and of hydrogenic ions by antiprotons is quite different from that for impact by positively charged particles at low energy. For atomic hydrogen these differences are due to the merging of the quasi-molecular electronic eigenenergies with the continuum, and the consequent shifting of the adiabatic regime to extremely low energies. For $\mathrm{He}^{+}$and other hydrogenic ions, the levels do not merge with the continuum, and the drop of the cross section at low energies experienced in positive particle impact is more closely obtained. However, the topology of the complex eigenenergy surface governing this low energy behavior is quite different from that in the positive particle case. TDSE and HC calculations have been compared here with recent experimental measurements and the results of other approaches yielding a detailed description of the ionization process. Three-body classical dynamics are shown to approximately describe the physics of the low 
energy ionization problem whereas CDW-EIS results grossly underestimate the magnitude of the ionization cross section.

In this light, recent measurements [6] for $p^{-}+H e$ which compared favorable at low energies with CDW-EIS, which could only be fortuitously correct in the low energy range, are difficult to interpret. In particular, the measurements dropped off rapidly at low energies even though recent calculations of the two-electron energy levels show that the ground state should be promoted very near the one-electron continuum (i.e. the $p^{-}+H e^{+}(1 s)$ molecular term) at small interparticle distances [5]. That result, and an earlier less complete calculation of these curves [10] seem in contradiction with the experimental findings. Clearly, further theoretical and experimental work is need to elucidate the behavior in this two-electron case.

The authors acknowledge the support of the US DOE, Offices of Fusion Energy and Basic Energy Sciences through Contract Number DE-AC05-84OR21400, managed by Lockheed Martin Energy Systems. JCW has been supported by the NSF. We also acknowledge helpful advice from P. Gavras, M. Pindzola, A. Salin, J. Burgdörfer, S. Ovchinnikov, G. Schiwietz, and R. Janev. 


\section{REFERENCES}

[1] D.R. Schultz, R.E. Olson, and C.O. Reinhold, J. Phys. B 24, 521 (1991); H. Knudsen and J.F Reading, Phys. Rep. 212, 107 (1992).

[2] L.H. Andersen et al. Phys. Rev. A 36, 3612 (1987); L.H. Andersen et al., Phys. Rev. Lett. 57, 2147 (1986); M. Charlton et al., J. Phys. B 21, L545 (1988).

[3] R.E. Olson and T.J. Gay, Phys. Rev. Lett. 61, 302 (1988).

[4] M. Agnello et al., Phys. Rev. Lett. 74, 371 (1995).

[5] G. Schiwietz et al. J. Phys. B submitted; private communication.

[6] P. Hvelplund et al., J. Phys. B 27, 925 (1994).

[7] H. Knudsen et al., Phys. Rev. Lett. 74, 4627 (1995).

[8] D.R. Schultz, Phys. Rev. A 40, 2330 (1989).

[9] E. Fermi and E. Teller, Phys. Rev 72, 399 (1947); O.H. Crawford, Proc. Phys. Soc. London 91, 279 (1967); I.V. Komarov et al., in Spheroidal and Coulomb spheroidal functions (Nauka, Moscow, 1976); I. Shimamura, Phys. Rev. A 46, 3776 (1992).

[10] M. Kimura and M. Inokuti, Phys. Rev. A 38, 3801 (1988).

[11] S.Yu. Ovchinnikov and J.H. Macek, AIP Conference Proceedings 274, VI International Conference on the Physics of Highly Charged Ions, p.622, New York (1992).

[12] V. Maruhn-Rezwani et al., Phys. Rev. Lett. 43, 512 (1979); C. Bottcher, Phys. Rev. Lett. 48, 85 (1982); K.C. Kulander et al., Phys. Rev. A 25, 2968 (1982); P. Gavras et al., Phys. Rev. A 52, 3868 (1995).

[13] J.C. Wells et al., Phys. Rev. A (1995) submitted.

[14] N. Toshima, Phys. Lett. A 175, 133 (1993).

[15] M.H. Matir et al., J. Phys. B 15, 1729 (1982). 
[16] J.S. Cohen, Phys. Rev. A 36, 2024 (1987).

[17] E.A. Solov'ev, Sov. Phys. JETP 54, 893 (1981); E.A. Solov'ev, Sov. Phys. Usp. 32, 228 (1989); T.P. Grozdanov and E.A. Solov'ev, Phys. Rev. A 42, 2703 (1990).

[18] L.D. Landau and E.M. Lifshitz, Quantum Mechanics: Non-Relativistic Theory, Pergamon, Oxford (1977).

[19] P.S. Krstić et al., J. Phys. B (1995) submitted.

[20] R.K. Janev et al., J. Phys. B 28, L615 (1995). 


\section{Figure Captions}

Figure 1. The electronic energy as a function of the internuclear separation for $p^{-}+H$ and $p^{-}+H e^{+}$.

Figure 2. The total ionization cross section as a function of collision energy for $p^{-}+H$. The experimental measurements [0] (squares) are compared with various theoretical approaches (CTMC - solid curve, TDSE - circles, CDW-EIS - long dashed curve, $\mathrm{CC}_{s}$ - solid curve connecting open circles, $\mathrm{CC}_{t}$ - dashed curve, $\mathrm{CC}_{m}$ - dot-dash curve), and the Fermi-Teller limit.

Figure 3. Position of the S-superseries in the complex plane of internuclear separation for various nuclear charges $(Z)$ of the hydrogenic ions.

Figure 4. The total ionization cross section as a function of collision energy for $p^{-}+H e^{+}$. The theoretical results are denoted by the solid curve (CTMC), circles (TDSE), long dashed curve (CDW-EIS), and dashed curve (hidden crossings (HC) theory). 